\title{
Moscas frugívoras e seus parasitoides nos municípios de Pelotas e Capão do Leão, Rio Grande do Sul, Brasil
}

\author{
Frugivorous flies and their parasitoids in the cities of Pelotas and Capão do Leão, \\ Rio Grande do Sul, Brazil
}

\author{
Adrise Medeiros Nunes ${ }^{*}$ Fernanda Appel Müller $^{\mathrm{I}}$ Rafael da Silva Gonçalves $^{\mathrm{I}}$ \\ Mauro Silveira Garcia ${ }^{\mathrm{I}}$ Valmir Antonio Costa ${ }^{\mathrm{II}}$ Dori Edson Nava ${ }^{\mathrm{III}}$
}

\section{RESUMO}

\begin{abstract}
As moscas frugívoras (Tephritoidea) são as principais pragas da fruticultura de clima temperado no Brasil. $O$ objetivo do presente trabalho foi avaliar a infestação desses dípteros e a ocorrência de seus parasitoides em frutíferas nos municípios de Pelotas e Capão do Leão, localizados na região Sul, nas safras agrícolas de 2007/08 e 2008/09. Foram coletados frutos de araçazeiro-amarelo e vermelho (Psidium cattleianum Sabine, 1821), butiazeiro [Butia capitata (Mart.) Becc., 1916], caquizeiro (Diospyros kaki Linnaeus, 1753), cerejeira-do-mato (Eugenia involucrata DC., 1828), goiabeira [Psidium guajava (Linnaeus, 1753)], goiabeira-serrana [Acca sellowiana (Berg.) Burret, 1941], nespereira [Eriobotrya japonica (Thunb.) Lindley, 1822], pessegueiro [Prunus persicae (L.) Batsch, 1801], pitangueira (Eugenia uniflora Linnaeus,1753) e uvalheira (Eugenia pyriformis Cambessèdes, 1832). Os frutos foram coletados e transportados para o laboratório, onde foram individualizados e determinados os seguintes parâmetros: índice de infestação das moscas, índice de parasitismo e frequência de indivíduos por espécie de parasitoide. Foram constatadas duas espécies de Tephritidae, Anastrepha fraterculus (Wiedemann, 1830) (90,5\%) e Ceratitis capitata (Wiedemann, 1824) (9,5\%) e duas de Lonchaeidae, Neosilba zadolicha McAlpine \& Steyskal, 1982 (87,8\%) e uma espécie ainda não descrita, referida como Neosilba $n$. sp. 3 (12,2\%). Anastrepha fraterculus é a espécie mais abundante nos dois municípios, sendo constatada na maioria das frutíferas coletadas. Caquizeiro e goiabeira foram os hospedeiros que apresentaram o maior índice de infestação por C. capitata. Quanto às espécies de Neosilba, a maior infestação ocorreu em frutos de goiabeira-serrana. Dos parasitoides emergidos, foram identificadas três espécies, sendo duas de Braconidae, Doryctobracon areolatus (Szépligeti, 1911) (52,6\%) e Opius
\end{abstract}

bellus (Gahan, 1930) (27,5\%) e uma espécie de Figitidae, Aganaspis pelleranoi (Brèthes, 1924) (20,0\%). Doryctobracon areolatus foi o parasitoide mais frequente na maioria das frutíferas amostradas, com exceção da pitangueira e cerejeirado-mato em que predominou $\boldsymbol{O}$. bellus, e em pessegueiro com predomínio de A. pelleranoi.

Palavras-chave: Anastrepha fraterculus, Ceratitis capitata, controle biológico, moscas-das-frutas.

\section{ABSTRACT}

Frugivorous flies (Tephritoidea) are the main pests in temperate-zone fruit crop in Brazil. The objective of this research is to evaluate the infestation of these flies and the incidence of their parasitoids in fruit trees from 2007/08 and 2008/9 crops in Pelotas and in Capão do Leão, located in the south of Rio Grande do Sul. Fruits were collected from yellow guava and red guava (Psidium cattleianum Sabine, 1821), pindo palm [Butia capitata (Mart.) Becc., 1916], persimmon (Diospyros kaki Linnaeus, 1753), wild cherry (Eugenia involucrata DC., 1828), guava [Psidium guajava (Linnaeus, 1753)], feijoa tree [Acca sellowiana (Berg.) Burret, 1941], medlar [Eriobotrya japonica (Thunb.) Lindley, 1822], peach [Prunus persicae (L.) Batsch, 1801], surinam cherry (Eugenia uniflora Linnaeus, 1753) and (Eugenia pyriformis Cambessèdes, 1832). The fruit was collected and transported to the laboratory, where the following parameters were individually determined: fly infestation rate, parasitism rate, and its frequency per parasitoid specie. It was found two species of Tephritidae, Anastrepha fraterculus (Wiedemann, 1830) (90.5\%) and Ceratitis capitata (Wiedemann, 1824) (9.5\%) and two of Lonchaeidae, Neosilba zadolicha McAlpine \& Steyskal, 1982 $(87.8 \%)$ and a species not yet described, called Neosilba n. $s p$.

'Departamento de Fitossanidade, Universidade Federal de Pelotas (UFPel), CP 354, 96010-900, Pelotas, RS, Brasil. E-mail: adrisenunes@gmail.com. *Autor para correspondência.

"Instituto Biológico, Agência Paulista de Tecnologia dos Agronegócios, Secretaria de Agricultura e Abastecimento, Campinas, SP, Brasil.

"IILaboratório de Entomologia, Embrapa Clima Temperado, Pelotas, RS, Brasil. 
$3(12.2 \%)$. Anastrepha fraterculus is the most abundant species in the two cities and it was detected in most fruit crops collected. The persimmon and the guava were the hosts that showed the highest index of $\boldsymbol{C}$. capitata infestation. Regarding to the species of Neosilba, the highest infestation occurred in fruits of feijoa tree. From the emerged parasitoids, it was identified three species two of Braconidae, Doryctobracon areolatus (Szépligeti, 1911) (52.6\%) and Opius bellus (Gahan, 1930) (27.5\%) and a species of Figitidae, Aganaspis pelleranoi (Brèthes, 1924) (20.0\%). Doryctobracon areolatus was the most frequent parasitoid in most sampled fruit trees, except for surinam cherry and wild cherry, where $\boldsymbol{O}$. bellus was predominant and for peach, $\boldsymbol{A}$. pelleranoi.

Key words: Anastrepha fraterculus, Ceratitis capitata, biological control, fruit flies.

\section{INTRODUÇÃO}

Na região Sul do Brasil, o Estado do Rio Grande do Sul (RS) é líder na produção de frutíferas de clima temperado, embora enfrente problemas com a ocorrência de insetos-praga, principalmente com as moscas frugívoras (Tephritoidea). Os danos causados por esses insetos contribuem para o aumento das perdas e dificultam as exportações de frutas, devido às barreiras quarentenárias impostas pelos países importadores (ZUCCHI, 2000).

Para a região Sul do Brasil, estudos de levantamento de espécies de moscas frugívoras são escassos, destacando-se os trabalhos de SALLES \& KOVALESKI (1990), HICKEL\& DUCROQUET (1993), SALLES (1995), GARCIA \& CORSEUIL (1998a,b), GARCIA et al. (2003a,b), SILVA et al. (2006) e ALBERTI et al. (2009). Esses estudos demonstram que Anastrepha fraterculus (Wiedemann, 1830) representa a espécie mais abundante na região. Além de $\boldsymbol{A}$. fraterculus, moscas pertencentes à Lonchaeidae são relatadas infestando frutos de importância econômica no Brasil e no RS desde a década de 30 , porém o conhecimento desse grupo de dípteros é escasso em todo o país (ARAÚJO \& ZUCCHI, 2002).

Os himenópteros das subfamílias Eucoilinae (Figitidae) e Opiinae (Braconidae) compõem os grupos de inimigos naturais mais importantes no controle de larvas de dípteros frugívoros (GUIMARÃES et al., 2003; NUNES et al., 2011). O conhecimento da ocorrência desses himenópteros nos pomares é importante para auxiliar no estabelecimento de estratégias de controle e fomentar futuros estudos visando ao controle biológico aplicado.

Nesse sentido, objetivou-se avaliar, no presente trabalho, a infestação de moscas frugívoras e a ocorrência de seus parasitoides em frutíferas nos municípios de Pelotas e de Capão do Leão, RS.

\section{MATERIAL E MÉTODOS}

O estudo foi realizado em pomares de frutíferas não comerciais nos municípios de Pelotas e Capão do Leão, RS, nas safras de 2007/08 e 2008/09. Em Pelotas, foram coletados frutos no pomar da Embrapa Clima Temperado (latitude 31 ${ }^{\circ} 40^{\prime} 48.48^{\prime \prime} \mathrm{S}$, longitude $52^{\circ} 26^{\prime} 42.71^{\prime \prime} \mathrm{O}$ e altitude de $175 \mathrm{~m}$ ) e, em Capão do Leão, no Banco Ativo de Germoplasma da Palma da Universidade Federal de Pelotas (UFPel) (latitude $31^{\circ} 48^{\prime} 08.62^{\prime \prime S}$, longitude 52³0'45.39"O e altitude de $198 \mathrm{~m}$ ).

Em ambos os locais, foram coletados frutos ao acaso, com sintomas de ataque de moscas frugívoras tanto na planta, quanto no solo, de acordo com a época de frutificação, compreendendo as seguintes espécies: araçazeiro-amarelo e vermelho (Psidium cattleianum Sabine, 1821), butiazeiro [Butia capitata (Mart.) Becc., 1916], caquizeiro (Diospyros kaki, Linnaeus, 1753), cerejeira-do-mato (Eugenia involucrata DC., 1828), goiabeira [Psidium guajava (Linnaeus, 1753)], goiabeira-serrana [Acca sellowiana (Berg.) Burret, 1941], nespereira [Eriobotrya japonica (Thunb.) Lindley, 1822], pessegueiro [Prunus persicae (L.) Batsch, 1801], pitangueira (Eugenia uniflora Linnaeus, 1753) e uvalheira (Eugenia pyriformis Cambessèdes, 1832). O tamanho das amostras foi variável, dependendo da disponibilidade de frutos no campo. Para a maioria das frutíferas, foram coletados 150 frutos em cada safra, com exceção de pitangueira, goiabeira e araçazeiro-vermelho, para os quais foram coletados 300, 115 e 100 frutos, respectivamente. As amostras foram devidamente identificadas (data e local), acondicionadas em caixas plásticas e transportadas para o Laboratório de Entomologia da Embrapa Clima Temperado.

Após, os frutos foram pesados e individualmente colocados em copos plásticos $(500 \mathrm{~mL})$ sobre uma camada de vermiculita fina. Em seguida, os copos foram cobertos com tecido tipo voile e mantidos em sala climatizada, sob condições controladas de temperatura $\left(25 \pm 2^{\circ} \mathrm{C}\right)$ e de umidade relativa $(70 \pm 10 \%)$. Próximo da pupação, a vermiculita foi peneirada diariamente e os pupários obtidos foram separados do substrato, quantificados, etiquetados e acondicionados em tubos de vidro transparentes, contendo vermiculita umedecida como substrato para emergência das moscas e/ou parasitoides. Os adultos das moscas ou dos parasitoides foram colocados em potes plásticos $(5 \mathrm{~mL})$ com álcool $70 \%$, para posterior identificação. A identificação das espécies de Anastrepha e de Ceratitis foi realizada utilizando-se chave dicotômica de ZUCCHI (2000). Em caso de dúvida, as moscas e os parasitoides foram identificados por especialistas dos grupos. 
Com os dados obtidos, foi determinado o índice de infestação das moscas, sendo calculado por meio de duas equações: (1) número médio de pupários por quilo de fruta fresca e (2) pelo número médio de pupários por fruto, de acordo com MALAVASI \& MORGANTE (1980). Para os parasitoides, foram determinados o índice de parasitismo $(\mathrm{P})$ e a frequência de indivíduos por espécie (E), de acordo com MATRANGOLO et al. (1998).

\section{RESULTADOS E DISCUSSÃO}

Foram obtidos 15.875 pupários do total de frutos, dos quais emergiram 12.070 dípteros frugívoros e 546 parasitoides. Do total de dípteros emergidos, 12.017 pertenceram à Tephritidae [90,5\% são espécimes de A. fraterculus e $9,5 \%$ de Ceratitis capitata (Wiedemann, 1824)] e 53 à Lonchaeidae, espécimes de Neosilba zadolicha Mcalpine \& Steyskal, 1982, e uma espécie ainda não descrita, referida como Neosilba n. sp. 3. As 12 fêmeas de lonqueídeos foram denominadas como Neosilba spp., já que a identificação desse inseto é baseada na análise das estruturas da genitália dos machos (MCALPINE \& STEYSKAL, 1982). Do total de 41 machos, $36(87,8 \%)$ foram identificados como $N$. zadolicha e cinco (12,2\%) como Neosilba n. sp. 3 .
$\mathrm{Na}$ maioria das espécies de frutíferas amostradas no pomar pertencente à Embrapa Clima Temperado, ocorreu A. fraterculus, com exceção de butiazeiro, em que não houve infestação. Nessa localidade, só houve registro de $\boldsymbol{C}$. capitata nos frutos de araçazeiro-amarelo. No Banco Ativo de Germoplasma da Palma, A. fraterculus também ocorreu na maioria das espécies de frutíferas amostradas com exceção de caquizeiro, no qual só foi constatada $\boldsymbol{C}$. capitata (67,37\% dos espécimes amostrados). Ceratitis capitata ocorreu também em frutos de goiabeira $(28,43 \%)$, em frutos de araçazeiro-amarelo $(3,41 \%)$ e em frutos de araçazeiro-vermelho (0,79\%). Nessa localidade, não houve a ocorrência de lonqueídeos. Anastrepha fraterculus foi a espécie mais abundante, infestando nove dos hospedeiros amostrados, só não sendo constatada em frutos de butiazeiro e caquizeiro.

$\mathrm{Na}$ maioria das frutíferas, o número de espécimes de $\boldsymbol{A}$. fraterculus foi sempre superior ao das demais espécies. O maior índice médio de infestação de A. fraterculus ocorreu em frutos de pessegueiro (Tabela 1). SALLES \& KOVALESKI (1990), em levantamento realizado no município de Pelotas, em pomares comerciais de pessegueiro e citros,

Tabela 1 - Moscas frugívoras e seus parasitoides, índices de infestação e de parasitismo, a partir de levantamentos realizados em frutíferas nas safras 2007/08 e 2008/09. Pelotas e Capão do Leão, RS.

\begin{tabular}{|c|c|c|c|c|c|c|}
\hline \multirow{2}{*}{ Frutíferas } & \multicolumn{3}{|c|}{ Moscas frugívoras } & \multicolumn{2}{|c|}{ Índice de Infestação } & \multirow{2}{*}{ Parasitismo $(\%)$} \\
\hline & A.f & C.c & $\mathrm{N}$ & $\mathrm{P} / \mathrm{F}^{-1}$ & $\mathrm{P} \mathrm{kg}^{-1}$ & \\
\hline Uvalheira $^{1}$ & $86 / 157 *$ & - & - & $0,91 / 1,17$ & $68,2 / 82,30$ & $0 / 4,85$ \\
\hline Araçazeiro-amarelo ${ }^{1}$ & $161 / 153$ & $0 / 7$ & - & $1,50 / 1,80$ & $101,4 / 116,30$ & $4,73 / 15,34$ \\
\hline Araçazeiro-vermelho ${ }^{1}$ & $136 / 484$ & - & - & $1,47 / 4,26$ & $180,5 / 368,20$ & $6,85 / 2,81$ \\
\hline Pitangueira $^{1}$ & $134 / 151$ & - & $5 / 5$ & $0,68 / 0,72$ & $129 / 164,20$ & $25,67 / 23,15$ \\
\hline Goiabeira serrana $^{1}$ & $166 / 173$ & - & $14 / 17$ & $1,49 / 1,60$ & $37,40 / 42$ & $1,64 / 2,06$ \\
\hline Goiabeira $^{1}$ & $756 / 464$ & - & - & $8,43 / 5,25$ & $98,30 / 64,10$ & $4,06 / 7,57$ \\
\hline Nespereira $^{1}$ & $301 / 313$ & - & $4 / 1$ & $3,29 / 3,51$ & $117,30 / 125,20$ & $0 / 3,08$ \\
\hline Pessegueiro $^{1}$ & $2901 / 2815$ & - & $0 / 2$ & $24,67 / 23,57$ & $354,40 / 261,50$ & $1,26 / 1,19$ \\
\hline Cerejeira-do-mato $^{1}$ & $132 / 148$ & - & $3 / 2$ & $2,18 / 2,31$ & $235,30 / 252$ & $31,12 / 31,51$ \\
\hline Araçazeiro-amarelo ${ }^{2}$ & $94 / 69$ & $19 / 13$ & - & $0,91 / 0,67$ & $57 / 42,30$ & $0 / 4,65$ \\
\hline Araçazeiro-vermelho ${ }^{2}$ & $21 / 27$ & $2 / 7$ & - & $0,29 / 0,40$ & $0,05 / 0,08$ & $8,00 / 10,53$ \\
\hline Caquizeiro $^{2}$ & - & $493 / 277$ & - & $4,03 / 2,12$ & $31,20 / 24,50$ & $0 / 0$ \\
\hline Goiabeira**2 & $417 / 615$ & $186 / 139$ & - & $4,73 / 5,89$ & $65,30 / 70,50$ & $3,52 / 7,82$ \\
\hline
\end{tabular}

\footnotetext{
* Valores separados pela barra indicam as duas épocas de coleta: 2007/08 e 2008/09.

** Parasitoides emergidos de frutos de goiabeira coletados no Capão do Leão (seis foram provenientes de $C$. capitata).

Local de coleta: Pelotas ${ }^{1}$; Capão do Leão ${ }^{2}$.
} 
constataram que $\boldsymbol{A}$. fraterculus representa a totalidade dos tefritídeos, coletados em ambos os pomares. Em outras regiões do RS, A. fraterculus também foi a principal espécie em pomares de pessegueiro no município de Porto Alegre (GARCIA \& CORSEUIL, 1998a) e em citros na região do Vale do Rio Caí (SILVA et al., 2006; GATTELLI et al., 2008).

Dentre as frutíferas infestadas por $\boldsymbol{C}$. capitata, constatou-se que o caquizeiro e a goiabeira foram os hospedeiros que apresentaram o maior índice de infestação. As menores infestações de $\boldsymbol{C}$. capitata ocorreram em frutos de araçazeiro-vermelho e de araçazeiro-amarelo (Tabela 1).

O lonqueídeo $N$. zadolicha foi constatado infestando os frutos de goiabeira-serrana. GATTELLI et al. (2008), em frutos do mesmo hospedeiro, encontraram exemplares de Neosilba n. sp. 3. Em pitangueira, foram identificadas $N$. zadolicha e Neosilba n. sp. 3 e, nos frutos de nespereira, pessegueiro e cerejeira-do-mato, todos os lonqueídeos eram fêmeas de Neosilba spp.
Dos 546 parasitoides emergidos, foram identificadas três espécies, sendo duas pertencentes a Braconidae, Doryctobracon areolatus (Szépligeti, 1911) (52,6\%) e Opius bellus (Gahan, 1930) (27,5\%), e uma espécie pertencente a Figitidae, Aganaspis pelleranoi (Brèthes, 1924) (20,0\%) (Tabela 2). Nas duas safras avaliadas, 2007/08 e 2008/09, nos municípios de Pelotas e Capão do Leão, D. areolatus foi o parasitoide predominante (51,5 e 53,6\%, respectivamente) e foi coletado na maioria das frutíferas amostradas, com exceção de pitangueira e cerejeira-do-mato, em que $\boldsymbol{O}$. bellus foi mais frequente, e, em pessegueiro, em que $\boldsymbol{A}$. pelleranoi foi o mais abundante (Tabela 2).

Em trabalho realizado por SALLES (1996) em Pelotas, D. areolatus também foi encontrado em diversas frutíferas parasitando larvas de $\boldsymbol{A}$. fraterculus. De acordo com MATRANGOLO et al. (1998), D. areolatus poderia antecipar o parasitismo das larvas com relação as outras espécies nativas, que parasitam larvas em estádios mais desenvolvidos, resultando em uma vantagem em relação as outras espécies. GARCIA

Tabela 2 - Valor absoluto e freqüência das espécies de parasitóides (E\% - entre parênteses) em frutíferas, realizado nas safras de 2007/08 e 2008/09 em Pelotas e Capão do Leão, RS.

\begin{tabular}{|c|c|c|c|}
\hline \multirow{2}{*}{ Frutíferas } & Doryctobracon areolatus & Opius bellus & Aganaspis pelleranoi \\
\hline & 2007/08 2008/09 & $2007 / 08$ 2008/09 & 2007/08 2008/09 \\
\hline Uvalheira $^{1}$ & $0 / * 8(100)$ & - & - \\
\hline Araçazeiro-amarelo ${ }^{1}$ & $8(100) / 20(69,0)^{* *}$ & - & $0 / 9(31,0)$ \\
\hline Araçazeiro-vermelho ${ }^{1}$ & $10(100) / 14(100)$ & - & - \\
\hline Pitangueira $^{1}$ & $13(27,1) / 15(31,9)$ & $29(60,4) / 27(57,5)$ & $6(12,5) / 5(10,6)$ \\
\hline Goiabeira serrana $^{1}$ & $3(100) / 4(100)$ & - & - \\
\hline Goiabeira $^{1}$ & $32(100) / 21(55,3)$ & - & $0 / 17(44,7)$ \\
\hline Nespereira $^{1}$ & $0 / 10(100)$ & - & - \\
\hline Pessegueiro $^{1}$ & $14(37,8) / 12(37,5)$ & - & $23(62,2) 20(62,5)$ \\
\hline Cerejeira-do-mato $^{1}$ & $13(21,3) / 11(15,9)$ & $41(67,2) / 53(76,8)$ & $7(11,5) 5(7,3)$ \\
\hline Araçazeiro-amarelo ${ }^{2}$ & $0 / 4(100)$ & - & - \\
\hline Araçazeiro-vermelho ${ }^{2}$ & $2(100) / 4(100)$ & - & - \\
\hline Caquizeiro $^{2}$ & - & - & - \\
\hline Goiabeira $^{2}$ & $19(86,4) / 50(78,1)$ & - & $3(13,6) 14(21,9)$ \\
\hline
\end{tabular}

* Valores separados pela barra indicam as duas épocas de coleta: 2007/08 e 2008/09.

** Números entre parênteses indicam valores em percentual.

Local de coleta: Pelotas ${ }^{1}$ Capão do Leão ${ }^{2}$. 
\& CORSEUIL (2004), no oeste do Estado de Santa Catarina, obtiveram nove espécies de parasitoides, sendo A. pelleranoi $(25,6 \%)$ a espécie mais comum na região, seguido dos braconídeos Doryctobracon brasiliensis (Szépligeti, 1911) (21,1\%) e D. areolatus $(18,6 \%)$. Em levantamento realizado na província de Entre Rios, no Nordeste da Argentina, OVRUSKI et al. (2008) identificaram quatro espécies de parasitoides: D. brasiliensis, Utetes anastrephae (Viereck, 1913), D. areolatus e A.pelleranoi. Exemplares pertencentes as duas últimas espécies foram as que apresentaram os maiores percentuais de parasitismo.

Opius bellus foi o segundo mais frequente, porém só foi encontrado em frutos de cerejeira-do-mato e pitangueira. Isso, provavelmente, devido ao reduzido tamanho do ovipositor desses indivíduos. De acordo com MATRANGOLO et al. (1998), frutos com maior espessura de casca não permitem que fêmeas de Opius spp. e de $\boldsymbol{U}$. anastrephae alcancem as larvas.

Aganaspis pelleranoi foi encontrado parasitando larvas em frutos de araçazeiro-amarelo, pitangueira, goiabeira, cerejeira-do-mato e pessegueiro. Nas duas safras, a maior percentagem de parasitismo foi encontrada em frutos de pessegueiro $(62,2 / 62,5 \%)$ (Tabela 2). SALLES (1996) e GUIMARÃES et al. (1999), em trabalho relacionando coletas em Pelotas, também constataram a presença de $\boldsymbol{A}$. pelleranoi e uma espécie de Odontosema. Esses autores constataram um percentual de parasitismo de A. pelleranoi em larvas de $\boldsymbol{A}$. fraterculus mais alto em cerejeira-do-mato $(28,21 \%)$, quando comparado a este trabalho, em que o percentual de parasitismo em frutos de cerejeira-domato foi de 11,5 e 7,3\% para as safras de 2007/08 e de 2008/09, respectivamente.

Os maiores índices de parasitismo foram obtidos em duas frutíferas de casca fina, lisa e tamanho pequeno: pitangueira $(25,67 / 23,15 \%)$ e cerejeira-domato $(31,12 / 31,51 \%)$ (Figura 1). SALLES (1996), na região de Pelotas, e MATRANGOLO et al. (1998), na Bahia, também encontraram os maiores índices de parasitismo em espécies de casca fina, lisa e tamanho pequeno, como em cerejeira-do-mato, araçazeiro e pitangueira. Segundo CANAL \& ZUCCHI (2000), larvas em frutos pequenos de pericarpo fino e mesocarpo raso são mais facilmente parasitadas. HICKEL (2002) observou que, em frutos com polpa fina, o nível de parasitismo tende a ser máximo, enquanto um nível reduzido de parasitismo tende a persistir nos frutos de polpa espessa. O mesmo foi observado por MARINHO et al. (2009), com as maiores percentagens de parasitismo ocorrendo nos frutos menores, como, por exemplo, pombeiro (Citharexylum myrianthum Cham). e pitangueira. $\mathrm{O}$ inverso foi observado nos frutos maiores, em que as percentagens de parasitismo foram menores. No presente trabalho, os menores índices de parasitismo também foram encontrados em frutos de casca grossa, além de pilosa ou não, como a goiabeiraserrana $(1,64 / 2,06 \%)$ e o pessegueiro $(1,26 / 1,19 \%)$ (Figura 1).

\section{CONCLUSÃO}

Em pomares de frutíferas nos municípios de Pelotas e Capão do Leão, Anastrepha fraterculus é a espécie mais abundante dentre as frutíferas amostradas, com maiores infestações em frutos de pessegueiro. Os maiores índices de infestação de Ceratitis capitata ocorreram em caquizeiro e goiabeira. Espécies pertencentes ao gênero Neosilba foram encontradas infestando pitangueira, goiabeira-serrana, nespereira, pessegueiro e cerejeira-do-mato. Dentre os parasitoides, Doryctobracon areolatus é mais frequente na maioria das frutíferas amostradas, com exceção de Opius bellus em pitangueira e cerejeira-domato e Aganaspis pelleranoi em pessegueiro.

\section{AGRADECIMENTOS}

À Dra. Keiko Uramoto, pesquisadora do Instituto de Biociências da Universidade de São Paulo, pela identificação dos tefritídeos. Ao MSc. Pedro Carlos Strikis, do Departamento de Parasitologia do Instituto de Biologia da Universidade Estadual de Campinas, pela identificação dos lonqueídeos. Ao Prof. Dr. Flávio Roberto Mello Garcia, da Universidade Federal de Pelotas, pela identificação dos parasitoides. Ao CNPq (Conselho Nacional de Desenvolvimento Científico e Tecnológico), pela bolsa de doutorado e de produtividade em pesquisa do primeiro e do último autor, respectivamente. 


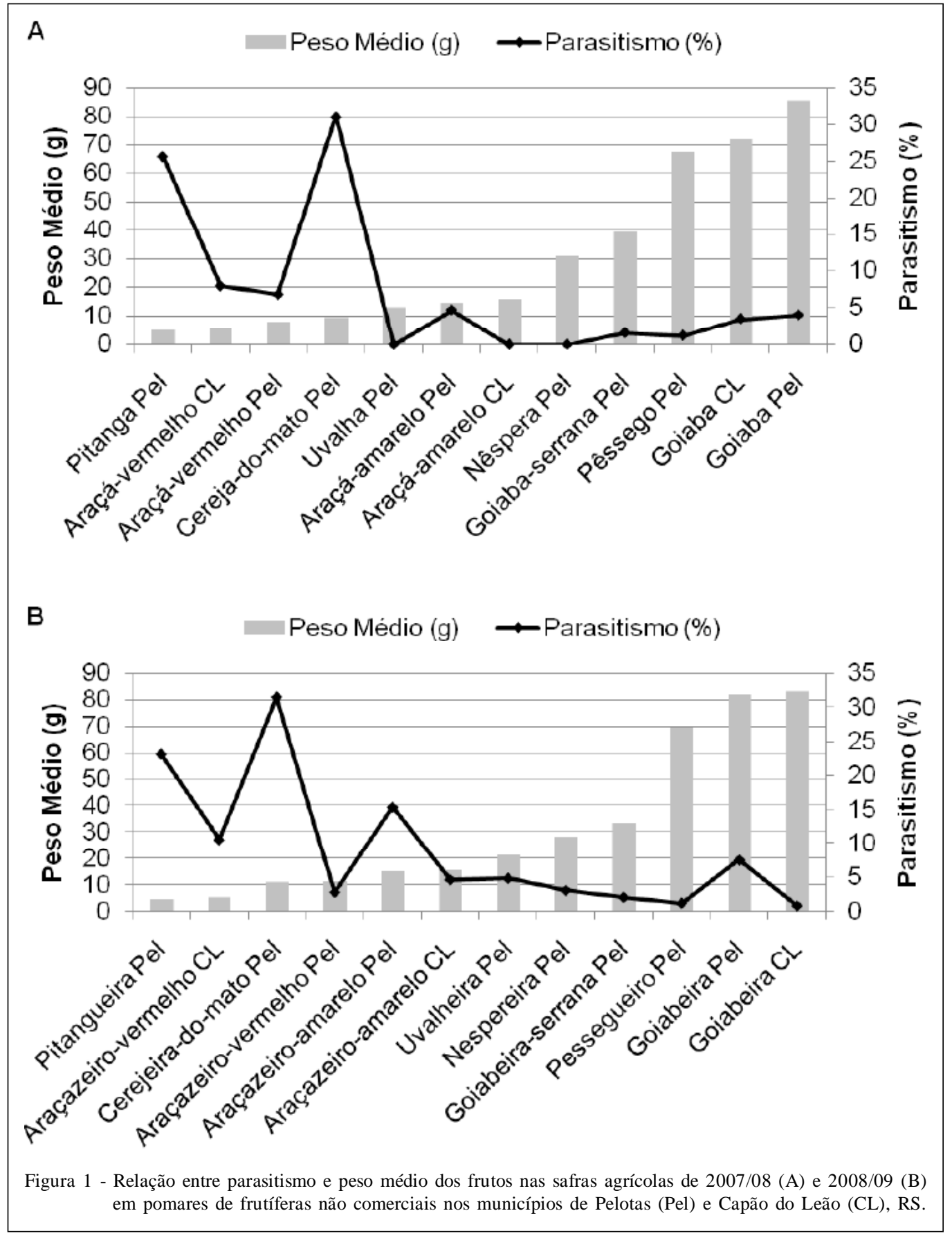

\section{REFERÊNCIAS}

ALBERTI, S. et al. Moscas-das-frutas em pomares de pessegueiro e maracujazeiro, no Município de Iraceminha, Santa Catarina, Brasil. Ciência Rural, v.39, n.5, p.1565-1568, 2009. Disponível em: <http://www.scielo.br/pdf/cr/v39n5/ a183cr654.pdf $>$. Acesso em: 5 jan. 2011. doi: 10.1590/S010384782009005000077 .

ARAÚJO, E.L.; ZUCCHI, R.A. Hospedeiros e níveis de infestação de Neosilba pendula (Bezzi) (Diptera: Lonchaeidae) na região de Mossoró/Assu, RN. Arquivos do Instituto Biológico, v.69, n.2, p. 91-94, 2002.

CANAL, N.A.; ZUCCHI, R.A. Parasitoides - Braconidae. In: MALAVASI, A.; ZUCCHI R.A. Moscas-das-frutas de importância econômica no Brasil: conhecimento básico e aplicado. Ribeirão Preto: Holos, 2000. p.199-126.

GARCIA, F.R.M.; CORSEUIL, E. Análise faunística de moscasdas-frutas (Diptera: Tephritidae) em pomares de pessegueiro em Porto Alegre, Rio Grande do Sul. Revista Brasileira de 
Zoologia, v.15, n.4, p.1111-1117, 1998a. Disponível em: <http://www.scielo.br/pdf/rbzool/v15n1/v15n1a13.pdf>. Acesso em: 24 dez. 2010. doi: 10.1590/S0101-81751998000400028.

GARCIA, F.R.M.; CORSEUIL, E. Flutuação populacional de Anastrepha fraterculus (Wiedemann) e Ceratitis capitata (Wiedemann) (Diptera: Tephritidae) em pomares de pessegueiro em Porto Alegre, Rio Grande do Sul. Revista Brasileira de Zoologia, v.15, n.1, p. 153-158, 1998b. Disponível em: <http:/ /www.scielo.br/pdf/rbzool/v15n1/v15n1a13.pdf>. Acesso em: 4 jan. 2011. doi: 10.1590/S0101-81751998000100013.

GARCIA, F.R.M.; CORSEUIL, E. Native hymenopteran parasitoids associated with fruit flies (Diptera: Tephritidae) in Santa Catarina State, Brazil. Florida Entomologist, v.87, n.4, p. 517-521, 2004. Disponível em: <http://journals.fcla.edu/ flaent/article/view/75349/>. Acesso em: 4 jan. 2011. doi: 10.1653/0015-4040(2004)087[0517:NHPAWF]2.0.CO;2.

GARCIA, F.R.M. et al. Análise faunística de espécies de moscasdas-frutas (Diptera: Tephritidae) na região Oeste de Santa Catarina. Neotropical Entomology, v.32, n.3, p.421-426, 2003a. Disponível em: <http://www.scielo.br/pdf/ne/v32n3/ 18754.pdf $>$. Acesso em: 4 jan. 2011. doi: 10.1590/S1519566X2003000300006.

GARCIA, F.R.M. et al. Flutuação populacional de Anastrepha fraterculus (Wiedemann, 1830) (Diptera: Tephritidae) na Região Oeste de Santa Catarina, Brasil. Revista Brasileira de Entomologia, v.47, n.3, p.415-420, 2003b. Disponível em: <http://www.scielo.br/pdf/rbent/v47n3/18905.pdf>. Acesso em: 5 jan. 2011. doi: 10.1590/S0085-56262003000300009.

GATTELLI, T. et al. Moscas frugívoras associadas a mirtáceas e laranjeira "Céu" na região do Vale do Rio Caí, Rio Grande do Sul, Brasil. Ciência Rural, v.38, n.1, p.236-239, 2008. Disponível em: <http://www.scielo.br/pdf/cr/v38n1/ a38v38n1.pdf>. Acesso em: 4 jan. 2011. doi: 10.1590/S010384782008000100038 .

GUIMARÃES, J.A. et al. Eucoilinae species (Hymenoptera: Cynipoidea: Figitidae) parasitoids of fruit-infesting dipterous larvae in Brazil: identity, geographical distribution and host associations. Zootaxa, v.278, p.1-23, 2003. Disponível em: <www.mapress.com/zootaxa/2003f/200278f.pdf>. Acesso em: 6 jan. 2011

GUIMARÃES, J.A. et al. Espécies de Eucoilinae (Hymenoptera: Cynipoidea: Figitidae) parasitoides de larvas frugívoras (Diptera: Tephritidae e Lonchaeidae) no Brasil. Anais da Sociedade Entomológica do Brasil, v.28, n.2, p.263-273, 1999. Disponível em: <http://www.scielo.br/pdf/aseb/v28n2/ v28n2a09.pdf $>$. Acesso em: 1 jan. 2011. doi: 10.1590/S030180591999000200009 .

HICKEL, E.R. Espessura da polpa como condicionante do parasitismo de moscas-das-frutas (Diptera: Tephritidae) por Hymenoptera: Braconidae. Ciência Rural, v.32, n.6, p.10051009, 2002. Disponível em: <http://www.scielo.br/pdf/cr/ v32n6/12746.pdf>. Acesso em: 1 jan. 2011. doi: 10.1590/ S0103-84782002000600014.

HICKEL, E.R.; DUCROQUET, J.P.H.J. Flutuação populacional de espécies de Anastrepha (Diptera: Tephritidae) relacionada com a fenologia de frutificação de pêssego e ameixa em Santa
Catarina. Anais da Sociedade Entomológica do Brasil, v. 22, n. 3, p. 591-596, 1993.

MALAVASI, A.; MORGANTE J.L. Biologia de "moscas-dasfrutas" (Diptera: Tephritidae). II Índices de infestação em diferentes hospedeiros e localidades. Revista Brasileira de Biologia, v.40, p.17-24, 1980.

MARINHO, C.F. et al. Parasitoides (Hymenoptera: Braconidae) de moscas-das-frutas (Diptera: Tephritidae) no estado de São Paulo: plantas associadas e parasitismo. Neotropical Entomology, v.38, n.3, p.321-326, 2009. Disponível em: <http://www.scielo.br/pdf/ne/v38n3/a04v38n3.pdf>. Acesso em: 6 jan. 2011. doi: 10.1590/S1519-566X2009000300004.

MATRANGOLO, W.J.R. et al. Parasitoides de moscas-dasfrutas (Diptera: Tephritidae) associados a fruteiras tropicais. Anais da Sociedade Entomológica do Brasil, v.27, n.4, p.593-603, 1998. Disponível em: <http://www.scielo.br/pdf/ aseb/v27n4/v27n4a12.pdf >. Acesso em: 5 jan. 2011. doi: 10.1590/S0301-80591998000400012.

MCALPINE, J.F.; STEYSKAL, G.C. A revision of Neosilba McAlpine with a key to the world genera of Lonchaeidae (Diptera). Canadian Entomologist, v.114, p.105-137, 1982.

NUNES, A.M. et al. Biology and parasitic potencial of Doryctobracon areolatus on Anastrepha fraterculus larvae. Pesquisa Agropecuária Brasileira, v.46, n.6, p.669-671, 2011. Disponível em: <http://www.scielo.br/pdf/pab/v46n6/ a14v46n6.pdf>. Acesso em: 24 jun. 2011. doi.org/10.1590/ S0100-204X2011000600014.

OVRUSKI, S.M. et al. Natural ocurrence of hymenopterous parasitoids associated with Anastrepha fraterculus (Diptera: Tephritidae) in myrtaceae species in Entre Rios, Northeastern Argentina. Florida Entomologist, v.91, n.2, p.220-227, 2008. Disponível em: <http://journals.fcla.edu/flaent/article/view/ 75792/73450>. Acesso em: 6 jan. 2011. doi: 10.1653/00154040(2008)91[220:NOOHPA]2.0.CO;2.

SALLES, L.A.B. Parasitismo de Anastrepha fraterculus (Wiedemann) (Diptera: Tephritidae) por Hymenoptera, na região de Pelotas, RS. Pesquisa Agropecuária Brasileira, v.31, n.11, p.769-774, 1996.

SALLES, L.A.B. Bioecologia e controle da mosca-dasfrutas sul-americana. Pelotas, RS, Embrapa: CPACT, 1995. $58 \mathrm{p}$.

SALLES, L.A.B.; KOVALESKI, A. Moscas-das-frutas em macieira e pessegueiro no Rio Grande do Sul. Horti Sul, v.1, n.3, p.5-9, 1990 .

SILVA, F.F. et al. Diversity of flies (Diptera: Tephritidae and Lonchaeidae) in organic citrus orchards in the Vale do Rio Caí, Rio Grande do Sul, Southern Brazil. Neotropical Entomologist, v.35, n.5, p.666-670, 2006. Disponível em: <http://www.scielo.br/pdf/ne/v35n5/15.pdf>. Acesso em: 6 jan. 2011. doi: 10.1590/S1519-566X2006000500015.

ZUCCHI, R.A. Taxonomia. In: MALAVASI, A.; ZUCCHI, R.A. Moscas-das-frutas de Importância Econômica no Brasil: conhecimento básico e aplicado. Ribeirão Preto: Holos, 2000. p.13-24. 